\title{
Tasa de importación como determinante en la decisión de compra del consumidor dentro del comercio electrónico
}

\author{
Import tax as a determining factor in the consumer's purchase decision within \\ electronic commerce
}

Richard García Alay

Christian Bermúdez Gallegos

Paul Murillo Delgado

Fecha de recepción: 6 de noviembre del 2018 


\title{
Tasa de importación como determinante en la decisión de compra del consumidor dentro del comercio electrónico
}

\author{
Import tax as a determining factor in the consumer's purchase decision within \\ electronic commerce
}

Richard García Alay ${ }^{1}$, Christian Bermúdez-Gallegos ${ }^{2}$, Paúl Murillo Delgado ${ }^{3}$

Como citar: García, R., Bermúdez, C., Murillo, P., (2019). Tasa de importación como determinante en la determinante en la decisión de compra del consumidor dentro del comercio electrónico, Revista Universidad de Guayaquil. 128(1), 12-19. DOI: https://doi.org/10.53591/rug.v128i1.1387

\section{RESUMEN}

El comercio electrónico en Ecuador mantiene un crecimiento sostenido en las dos últimas décadas gracias a los avances de las TICs. Sin embargo, el comportamiento del consumidor puede afectarse ante la imposición de tasas tributarias en las transacciones realizadas. El propósito de este estudio es determinar el efecto que representa una tasa de impuesto a la importación en la decisión de compra del consumidor online. Para esto, se consideró una metodología cuantitativa, partiendo de un modelo conceptual y contrastando con los datos tomados de una muestra aleatoria de 380 personas que realizan consumos dentro del comercio electrónico. Con alcance descriptivo se determinó el perfil del consumidor, y aplicando análisis correlacional se observó la fuerza de relación entre las variables de estudio. Los resultados demuestran que los consumidores online se inclinan más por comprar ropa y calzado, además el género femenino se ve más afectado a la imposición de tasas de importación en sus transacciones.

PALABRAS CLAVE: Tasa de importación, comportamiento de compra, consumidor online

\section{ABSTRACT}

Electronic commerce in Ecuador maintains sustained growth in the last two decades thanks to the advances of ICTs. However, consumer behavior may be affected by the imposition of tax rates on transactions carried out. The purpose of this study is to determine the effect of an import tax rate on the online consumer's purchasing decision. For this, a quantitative methodology was considered,

\footnotetext{
1Máster Universitario en Análisis y Visualización de Datos Masivos/ Visual Analytics and Big Data . Investigador Independiente, Ecuador. Correo electrónico: rgarciaalay@gmail.com

2 Magister en Informática de Gestión y Nuevas Tecnologías. Universidad de Guayaquil, Ecuador. Correo: christian.bermudezg@ug.edu.ec. ORCID: https://orcid.org/my-orcid?orcid=0000-0003-2594-5046

3 Magister en Administración de Empresas. Investigador independiente, Ecuador. Correo electrónico: paulmurillo@yahoo.com . ORCID: https://orcid.org/0000-0002-0819-1724
} 
starting from a conceptual model and contrasting with the data taken from a random sample of 380 people who consume within electronic commerce. With a descriptive scope, the consumer profile was determined, and applying correlational analysis the strength of the relationship between the study variables was observed. The results show that online consumers are more inclined to buy clothes and footwear, and the female gender is more affected by the imposition of import taxes on their transactions.

Keywords: Import tax; consumer's behavior; e-consumers.

\section{INTRODUCCIÓN}

El Internet es un medio que ha revolucionado la manera de realizar los negocios en el mundo empresarial dando lugar a lo que actualmente se denomina e-commerce o comercio electrónico, que se lo define como cualquier transacción comercial electrónica o intercambio de información para llevar a cabo un negocio (McLaren y McLaren, 2000).

El Internet se ha convertido en un medio que tienen las empresas para llegar a sus clientes y de esta forma obtener beneficios significativos los que se observan como una tendencia exponencial del gasto realizado en comercio electrónico, se puede decir que entre las ventajas está la comodidad y facilidad que implica hacer compras independiente de la distancia y el tiempo, además el poder recibir el producto en el domicilio además la facilidad de comparar productos, ofertas y descuentos dada la variedad de búsqueda e información (Constantinides, 2004). En Latinoamérica el crecimiento promedio es del $47 \%$ dada la expansión de la cobertura del internet por las ventajas en acceso y rapidez del servicio a más de la seguridad de parte de los consumidores (América Economía, 2012).

En países latinoamericanos, este crecimiento puede verse afectado por la imposición de tasas o aranceles aplicados al envío de paquetes desde el extranjero. En el caso de Ecuador, estas transacciones han sido reguladas por la última resolución tomada por el Comité de Comercio Exterior en Julio del 2014, mediante tasas impositivas y aranceles de acuerdo a seis categorías A, B, C, D, E, y F. La categoría B aplicada a las personas naturales, es la de mayor crecimiento y contempla un arancel de $\$ 42$ por importación de todos los paquetes que pesan hasta 4 kilogramos y hasta $\$ 400$ (Servicio Nacional de Aduana del Ecuador [SENAE], 2014). Tal resolución ha provocado que ciertos couriers detengan sus operaciones de servicio de tráfico postal debido a la reducción de sus utilidades según la Asociación Ecuatoriana de Correos Privados. 
En general el comportamiento de compra está relacionado con dos temas el precio y el nivel de conocimiento de los precios que poseen los compradores (Diaz \& Villarejo-Ramos, 2007). Es decir, la aplicación de una tasa de impuesto en el proceso de compra de un producto extranjero a través de Internet puede impactar en el comportamiento del consumidor online, ya que su nivel de ingresos es un factor relevante al momento de la decisión de compra (Bigné \& Ruiz, 2005), como también la percepción de utilidad y seguridad (Zubirán y López, 2009).

Otros autores han propuesto modelos de factores para explicar el comportamiento del consumidor online, entre ellos están Ruíz y Sanz (2006) en un estudio realizado en España con más de 3000 encuestas, se determinó como razón primordial para comprar por medio del Internet es la comodidad, además del precio y optimización de tiempo y ahorro de dinero, sin olvidar que la exposición al medio de Internet y la experiencia en su uso, también son factores importantes a considerar. Los autores afirmaron que existe relación entre las motivaciones utilitarias y el volumen de gasto. Por su parte, Zubirán y López (2009) en un estudio cuantitativo realizado en México consideraron las aspectos psicosociales y culturales como variables que afectan directamente el comportamiento de las personas en función de las innovaciones y avances tecnológicos. En Ecuador, Garzón (2014) determinó que los factores de edad, ingresos, peso y estado civil influyen en el comportamiento del consumidor online, como también la seguridad del sitio web, ayuda ofrecida, la calidad y el servicio de envío o correo postal, los cuales aseguran la lealtad del cliente y garantizan una experiencia satisfactoria.

Por conveniencia el estudio se lo realizó con la población de ciudadanos de la ciudad de Guayaquil, pues es una de las ciudades en donde se concentra el mayor volumen de las compras realizadas vía Internet en el Ecuador, con rango de edad comprendida entre los 20 y 40 años, por ser el grupo etario con mayor uso de internet, computadores y smartphones (Instituto Nacional de Estadísticas y Censos [INEC], 2011).

En base a lo expuesto el objetivo principal de la investigación fue confirmar si el impuesto de importación es un factor que determina el comportamiento de compra del consumidor online, para lo cual se estableció un conjunto de factores iniciales (Bigné \& Ruiz, 2005) incluyendo la variable impuesto que son los que determinan la decisión de compra online. Como objetivos secundarios, primero se estableció que la variable impuesto sea un determinante del comportamiento del consumidor online, y segundo se determinó la relación del impuesto con los demás factores que determinan tal comportamiento, lo cual fue verificado estadísticamente con el grado de dependencia entre las mismas. 


\section{MATERIALES Y MÉTODOS}

La naturaleza de esta investigación es cuantitativa por partir de un marco teórico proveniente de la exhaustiva revisión de la literatura, planteamiento de una hipótesis inicial para confirmar que el impuesto es un factor determinante del comportamiento de compra del consumidor online. El alcance de la investigación fue de tipo descriptivo correlacional puesto que se partió de resultados de estudios previos para explicar el grado de relación de las variables de estudio en el contexto ecuatoriano. La lógica de tipo deductiva ayudó a validar las hipótesis de estudio a partir de un análisis teórico. El estudio tuvo un corte transversal puesto que la recolección de datos se lo realizó en un periodo determinado de tiempo y de tipo no experimental ya que no se sometió a los encuestados a situaciones específicas ni se hubo manipulación de las variables de estudio (Hernández et al., 2006).

La literatura refleja que los factores que determinan en el comportamiento del consumidor online son: género, nivel de formación, nivel de ingresos del consumidor, frecuencia de exposición al medio de Internet, nivel de conocimiento del manejo de Internet y la variable compra que se refiere a métodos de pago, logística, seguridad y gasto asignado (Bigné \& Ruiz, 2005). El modelo conceptual propuesto por los autores considera que la exposición al medio y nivel de conocimiento de Internet son mediadoras de la decisión de compra. Así mismo existen otras variables demográficas que serán incluidas en el instrumento de medición como edad, estado civil, sector de residencia, además de los gustos y preferencias (Garzón, 2014).

Para la recolección de los datos se aplicó una encuesta previamente validada mediante una muestra piloto y con un grupo de expertos en comercio electrónico para validar los indicadores y redacción de las preguntas. La población de estudio la conformaron los consumidores online de la ciudad de Guayaquil comprendidos entre 20 y 40 años de edad, obteniendo una muestra de 384 personas mediante la fórmula para el cálculo de tamaño de la muestra de poblaciones grandes, con el 95\% de nivel de confianza. Se empleó muestreo aleatorio simple en varios sectores de la ciudad de Guayaquil en un solo periodo de tiempo del año 2015, un año después de aplicada la resolución de la tasa impositiva por parte del estado.

Luego los datos fueron analizados mediante estadística descriptiva para obtener las características del perfil del consumidor online y luego análisis multivariado donde se miden las relaciones entre las variables de estudio con la variable impuesto. El análisis multivariado se lo realizó con la herramienta estadística Infostat y empleando tablas de contingencia para determinar que variables tienen relación 
con el impuesto, y después análisis de correspondencia para reducir el número de dimensiones considerando que se pierda la menor cantidad de información posible (Closas, Zening, Amarilla \& Jovanovich, 2013).

\section{RESULTADOS Y DISCUSIÓN}

Los resultados del análisis descriptivo de los datos muestran que la aplicación de una tasa de impuesto en el envío de paquetes afecta negativamente al $85 \%$ de la población de consumidores online, los cuales dejaron de comprar o compran menos que antes de la aplicación de la tasa de $\$ 42$. El perfil del consumidor es de sexo masculino, soltero de 20 a 24 años, que vive en el sector norte de la ciudad, con estudios universitarios e ingreso promedio menor a $\$ 1000$, su acceso a internet es diario y compra con su tarjeta de crédito en tienda online cada 6 meses con preferencia a la ropa y zapatos.

El análisis multivariado realizado en dos etapas muestra en su primera etapa, realizado con tablas de contingencia, que la variable impuesto guarda relación significativa con el género, nivel de ingresos del consumidor, el sector de residencia del consumidor online, compras 2014 y compras 2015. Como se muestra en la Tabla 1, los valores calculados de chi $^{2}$ son mayores que sus valores críticos y los valores p menores a 0.05 como nivel de significancia, lo que indica que se rechaza la hipótesis nula y concluyendo que existe relación significativa entre las variables de estudio con el impuesto.

Tabla 1

Resultados de las variables que guardan relación significativa con la variable impuesto

\begin{tabular}{lllll}
\hline & $\begin{array}{l}\text { Estadístico } \\
\text { prueba chi }\end{array}$ & Grados de libertad & Valor $p$ & $\begin{array}{l}\text { Valor } \\
\text { crítico chi }^{2}\end{array}$ \\
\hline Género & 11 & 3 & 0.0117 & 7.81 \\
Nivel de Ingresos & 26.74 & 12 & 0.0084 & 21.03 \\
Sector de Residencia & 30.06 & 9 & 0.0004 & 16.92 \\
Compra 2014 & 28.02 & 15 & 0.0214 & 24.99 \\
Compra 2015 & 366.54 & 18 & 0.0001 & 28.87 \\
\hline
\end{tabular}

Del análisis de correspondencia simple de la variable impuesto con respecto al género del consumidor se obtuvo que el tipo masculino con un valor $\mathrm{chi}^{2} \mathrm{de} 6.27 \mathrm{y}$ el femenino con un valor $\mathrm{chi}^{2} \mathrm{de} 4.73$. El autovalor de la dimensión1 es de 0.19 con una inercia del $100 \%$ y la dimensión 2 tiene un autovalor nulo con una inercia del $0 \%$. Las coordenadas respectivas en la dimensión1 son para el género masculino de 0.22 y para el género femenino de -0.17. En cambio, para el análisis de correspondencia 
múltiple se muestran dos dimensiones cuyo autovalor es de 0.66 y $10.26 \%$ de inercia para la dimensión principal y 0.63 con inercia de $9.58 \%$ para la dimensión secundaria.

\section{Discusión}

Del análisis de correspondencia simple de la variable impuesto con respecto al género del consumidor se obtuvo que el tipo masculino es el que más contribuye al estadístico chi ${ }^{2}$ con 6.27 frente a 4.73 del femenino. Además, al reducir a una dimensión esta variable bidimensional se obtiene que un solo eje pueda capturar más información, con una inercia de 0.04 frente a una inercia nula del segundo eje. El gráfico de correlación biplot de un solo eje indica que el género femenino es el que sintió más el efecto negativo del impuesto en su comportamiento de compra, resultado un estado de "ya no compro" o "compro menos que antes". A diferencia del género masculino que "compra igual que antes" es decir no le afectado significativamente el impuesto aplicado al envío de paquetes.

En cambio, el análisis de correspondencia múltiple da lugar a interpretar las asociaciones entre las categorías de determinada variable cualitativa en base a una muestra definida (Molina, 2008), se la utilizó para analizar en conjunto las relaciones entre las variables impuesto, nivel de ingreso del consumidor, sector de residencia, compras. Luego de reducir una dimensión se obtuvieron dos dimensiones que captan el $19.84 \%$ de la información, el eje principal con una inercia de $10.96 \%$ y el eje secundario con inercia del $9.58 \%$. Con estos dos ejes se graficaron las coordenadas obtenidas de cada indicador que componen las variables obteniendo que: (a) las personas que les afectó el impuesto y que "ya no compran" tienen ingresos de \$700 a \$900 y viven en la zona periférica de Guayaquil; (b) el grupo que "compra igual que antes" tienen ingresos de $\$ 354$ a $\$ 1000$ y viven en el sector norte y sur de la ciudad, su promedio de compra en el 2014 fue de $\$ 180$ frente a $\$ 80$ para el 2015; (c) los que "compran menos que antes" corresponden al sector centro de la ciudad con ingresos superiores a los \$1000, su promedio de compra en el 2014 fue de \$300 a \$700 en comparación al 2015 que fue de $\$ 100$ a $\$ 300$; y (d) finalmente el grupo que compra "más que antes" es un grupo que en el 2014 su compra promedio era superior a $\$ 900$ y en el 2015 superior a $\$ 500$, no definen un ingreso y sector específico.

\section{CONCLUSIÓN}

Luego de las pruebas estadísticas se rechaza la hipótesis nula planteada por lo tanto se acepta la hipótesis alternativa que indica que existe influencia de la tasa de impuesto en el comportamiento de compra del consumidor online de la ciudad de Guayaquil. Es decir, la variable impuesto es decisiva 
en el comportamiento de compra, la mayoría de personas de la población respondió que compra menos luego de aplicarse la tasa de \$42 para el envío de paquetes desde el extranjero.

Descriptivamente el consumidor online guayaquileño es de estado civil soltero de edad comprendida entre 20 a 24 años, cursa sus estudios universitarios, y posee ingresos promedios entre $\$ 354$ a $\$ 1000$ y vive en el sector norte de la ciudad, su método de pago es con tarjeta de crédito con preferencia de compra en ropa y calzado.

El análisis multivariado demuestra que la aplicación de una tasa de impuesto tiene efecto negativo en el comportamiento de compra del consumido online de Guayaquil, afectando mayormente al género femenino, con residencia en el sector norte y sur de la ciudad, cuyo promedio en compra se vio disminuido en $\$ 100$ en relación con cuando no existía el impuesto. Las variables que son significativamente dependientes del impuesto son el género, nivel de ingresos del consumidor, el sector de residencia del consumidor online, y la variable compras.

Se recomienda emplear métodos estadísticos confirmatorios para afirmar los factores principales encontrados y sus relaciones de dependencia. Así mismo replicar este estudio en otros países para verificar la consistencia de los resultados obtenidos y poder generalizar el modelo de factores para contextos similares.

\section{REFERENCIAS}

América Economía Intelligence. (2012). El estudio de comercio electrónico en América Latina 2012. Obtenido de http://especiales.americaeconomia.com/2012/comercio-electronico-america-latina2012/graficos.php

Bigné, J., \& Ruíz, C. (2005). Antecedentes de la deción de compra en los entornos virtuales. Revista Europea de Dirección y Economía de la Empresa, 141-158.

Closas, A. H., Arriola, E. A., Zening, K., Isabel, C., Amarilla, M. R., \& Jovanovich, E. C. (2013). Análisis multivariante, conceptos y aplicaciones en psicología educativa y psicometría. Enfoques, 25(1), 6592

Constantinides, E. (2004). Influencing the online consumer's behavior: the Web experience. Internet research, $14(2), 111-126$.

Díaz, I. M. R., \& Villarejo-Ramos, Á. F. (2007). Comportamiento del consumidor ante los precios promocionales: una experiencia empírica. In Conocimiento, innovación y emprendedores: camino al futuro (p. 152). Universidad de La Rioja

Garzón, M. (2014). Propuesta de un modelo conceptual sobre los factores determinantes para la compra online de los consumidores ecuatorianos en el comercio electrónico B2C. Guayaquil. 
REVISTA UNIVERSIDAD DE GUAYAQUIL Vol. 128 No. 1 - 2019 (enero-junio)

ISSN: 2477-913X e-ISSN: 2806-5751

Hernández, R., Fernández, C., \& Baptista, P. (2006). Metodología de la investigación. México D.F: McGrawHill.

Instituto Nacional de Estadístico y Censos. (2011). Encuenta Nacional de Empleo y Subempleo. Obtenido de http://www.inec.gob.ec/sitio_tics/infografia.pdf

McLaren, C. H., \& McLaren, B. J. (2000). E-commerce: Business on the Internet. South-Western Educational Pub.

Ruíz, M., \& Sanz, B. (2006). Influencia de las motivaciones en la decisión de compra y en la lealtad hacia el Internet. Investigaciones Europeas de Dirección y Economía de la Empresa, 195-215.

Servicio Nacional de Aduana del Ecuador. SENAE (2014). El SENAE informa sobre el sistema de paquetería 4x4. Obtenido de http://www.aduana.gob.ec/contents/nov/press_room_view.jsp?idx=60

Zubirán, R., \& López, J. (2009). Factores críticos que determinan la intención de compra en línea en el comercio electrónico mexicano. Innovaciones de negocios, 237-256. 